\title{
Antimalarial prescribing and dispensing practices in health centres of Khartoum state, 2003-04
}

A.A. Mannan, ${ }^{1}$ E.M. Malik ${ }^{2}$ and K.M. Ali ${ }^{3}$

$$
\begin{aligned}
& \text { محارسات وصف وصرف الأدوية المضادَّة للمالاريا لدى مقدّمي الرعاية الصحية في المراكز الصحية بولاية الخرطوم }
\end{aligned}
$$

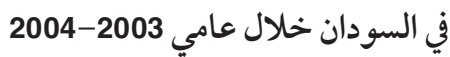

$$
\begin{aligned}
& \text { عبير أبو زيد عطا المنان، الفاتح محمد مانك مالك، كامل ميرغني علي }
\end{aligned}
$$

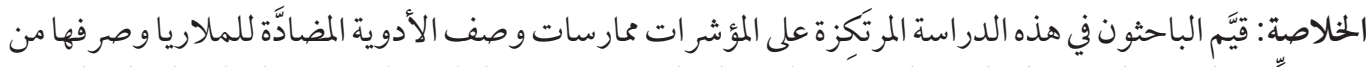

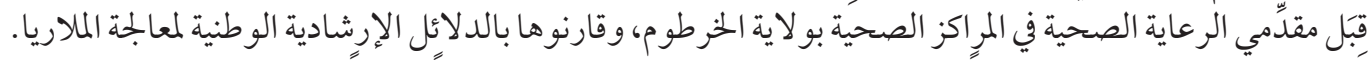

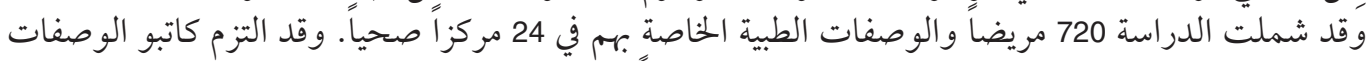

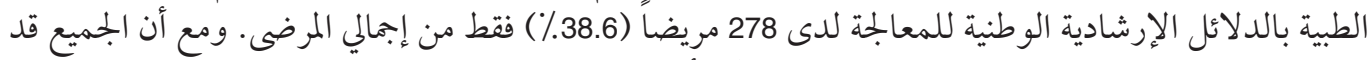

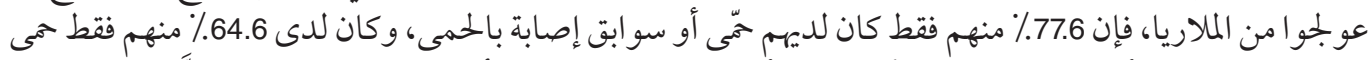

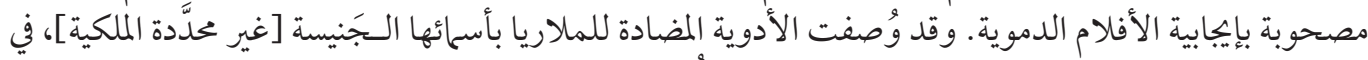

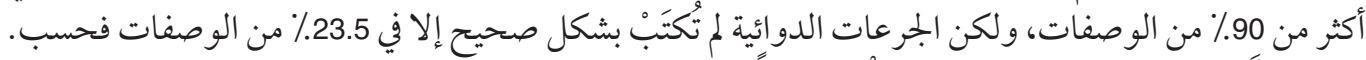

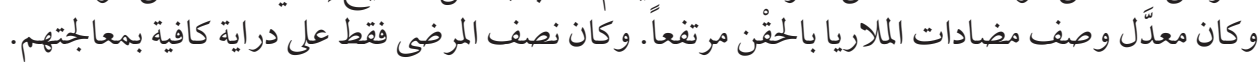

ABSTRACT This indicator-based study assessed the antimalarial drug prescribing and dispensing practices of health care providers in health centres of Khartoum state, and compared these with national guidelines for malaria treatment. A total of 720 patients and their prescriptions in 24 health centres were included. Prescribers adhered to national treatment guidelines for only 278 (38.6\%) of patients. Although all were treated for malaria, only $77.6 \%$ patients had fever or history of fever and only $64.6 \%$ had fever and positive blood films. More than $90 \%$ of prescriptions prescribed antimalarial drugs by generic names but dosage forms were correctly written in only $23.5 \%$. There was a high rate of prescribing antimalarial injections. Only half the patients had adequate knowledge of their treatment.

Pratiques en matière de prescription et d'administration d'antipaludéens dans les centres de santé de l'État de Khartoum, 2003-2004

RÉSUMÉ Cette étude fondée sur des indicateurs a évalué les pratiques en matière de prescription et d'administration d'antipaludéens chez les soignants exerçant dans les centres de santé de l'État de Khartoum, et les a comparées aux recommandations nationales relatives au traitement du paludisme. Au total, l'étude a porté sur 720 patients de 24 centres de santé et sur les prescriptions qui leur avaient été faites. Les prescripteurs suivaient les recommandations nationales pour seulement 278 (38,6\%) patients. Même si tous étaient traités contre le paludisme, seuls $77,6 \%$ d'entre eux avaient de la fièvre ou des antécédents de fièvre et $64,6 \%$ avaient de la fièvre et des étalements sanguins positifs. Sur plus de $90 \%$ des ordonnances, les antipaludéens étaient désignés par leur nom générique, mais la forme galénique était correctement indiquée sur seulement $23,5 \%$. Le taux de prescription d'antipaludéens par infection était très élevé. La moitié des patients seulement comprenaient bien à quoi correspondait leur traitement.

${ }^{1}$ National Malaria Control Programme; ${ }^{2}$ Roll Back Malaria Programme, Federal Ministry of Health, Khartoum, Sudan (Correspondence to A.A. Mannan: abeeratta@hotmail.com).

${ }^{3}$ Department of Community Medicine, University of Khartoum, Khartoum, Sudan.

Received: 02/10/05; accepted: 17/08/06

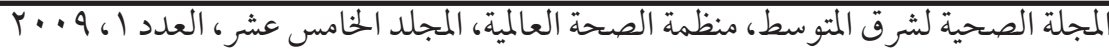




\section{Introduction}

Malaria is the major public health problem in Sudan. The whole country is considered endemic, ranging from holoendemic in the south to hypoendemic in the north. Plasmodium falciparum infection is overwhelmingly predominant $(90 \%)$ and Anopheles arabiensis is the main vector. About $40 \%$ of outpatient attendance is due to malaria, with a current estimated rate of 7-8 million cases, and 35 000-40 000 deaths per year due to malaria [1].

In many developing countries, including Sudan, inappropriate, ineffective and inefficient use of drugs commonly occurs at health facilities [2-4]. Lack of medicines, absence of updated guidelines and laboratory facilities and inadequate training combine to influence prescribing practices [5]. It is necessary to define prescribing patterns and to identify irrational prescribing habits in order to plan educational messages to prescribers [6].

Little is known about the determinants of poor use of antimalarial drugs. A recent review identified a scarcity of published literature on the subject [7]. Lack of reliable baseline data on antimalarial drug use in the health facilities in Sudan was one reason behind this study. The main objective of the study was to assess the antimalarial drug prescribing and dispensing practices of health care providers in health centres of Khartoum state, and to compare these practices with the national guidelines for malaria treatment.

\section{Methods}

\section{Setting}

Khartoum state includes the national capital of Sudan. Its population is 4568177 (estimated from the 1993 census), increasing by $4.04 \%$ per year. The population is a mixture of all Sudanese tribes and ethnic groups. Almost $68 \%$ of the population live in urban areas, $21 \%$ in rural areas and $11 \%$ are displaced people. The main health problems are tropical diseases: malaria is top of the list and is the major cause of death in children under the age of 5 years.

\section{Study design}

This was a facility-based, cross-sectional, descriptive, analytical study following World Health Organization (WHO) guidelines [8]. The sample size was calculated to be 720 patients using the standard crosssectional survey formula. Two-stage cluster sampling was used. The first stage was the selection of health centres using probability proportional to size and the second stage was the selection of patients. Accordingly the calculated sample was taken from 24 health centres (30 patients in each cluster).

\section{Data collection}

In each health centre, patients were selected as they emerged from the pharmacy after collecting their prescribed medicines. Four trained data collectors positioned themselves in the pharmacy and waited for patients. After interviewing patients, the information was recorded on a standard form (patient care form), then their prescriptions were collected and the data extracted were recorded on another form (prescribing indicator form). After the required number of patients had been interviewed, information on the types of antimalarial drugs available in stock and the presence of the national protocol poster in the health centre were recorded on a third form (facility summary form).

\section{Data management}

The information on each indicator was recorded and calculated for individual health centres. Data were entered, processed and analysed using SPSS, version 9.05. 


\section{Results}

The study sample comprised 720 patients who had been diagnosed as having uncomplicated malaria and had been prescribed antimalarial treatment by the health care providers in 24 health centres. However, $161(22.4 \%)$ patients had neither presented with fever nor reported an attack of fever within a few days prior to presentation. Moreover, only 378 patients $(52.5 \%)$ were physically examined. Thus the number correctly diagnosed as having malaria, i.e. patients who had both a history of fever and positive blood films for malaria, was only 465 (64.6\%). Examination of the prescriptions showed that less than $40 \%$ of patients were managed according to the recommended national treatment guidelines, i.e. had a history of fever, had positive blood films for malaria and were prescribed recommended antimalarial drugs. Prescriptions with the dosage form correctly written were found for only 169 patients $(23.5 \%)$ (Table 1).

Table 2 shows the pattern of prescribing indicators. Almost all the prescriptions (99.6\%) contained only 1 antimalarial drug and $659(91.5 \%)$ were prescribed in the ge-

Table 1 Complementary antimalarial drug use indicators in health facilities of Khartoum state ( $n=720$ prescriptions/patients)

\begin{tabular}{lcc}
\hline Indicator & No. & $\%$ \\
\hline Patients physically examined & 378 & 52.5 \\
Patients correctly diagnosed $^{\mathrm{a}}$ & 465 & 64.6 \\
$\begin{array}{l}\text { Prescriptions with dosage forms } \\
\quad \text { correctly written }\end{array}$ & 169 & 23.5 \\
$\begin{array}{l}\text { Patients managed according to } \\
\text { national treatment guidelines }\end{array}$ & 278 & 38.6 \\
\hline
\end{tabular}

aHistory of fever + positive blood films for malaria. ${ }^{b}$ History of fever + positive blood films for malaria + prescribed a recommended antimalarial drug.
Table 2 Antimalarial drug prescribing indicators in health facilities of Khartoum state ( $n=720$ prescriptions)

\begin{tabular}{lcc}
\hline Prescribing indicator & No. & $\%$ \\
\hline $\begin{array}{l}\text { Only } 1 \text { antimalarial drug } \\
\text { Antimalarial drug prescribed }\end{array}$ & 717 & 99.6 \\
$\quad$ by generic name & 659 & 91.5 \\
Antimalarial + antibiotic & 320 & 44.4 \\
Antimalarial + analgesic & 374 & 51.5 \\
Antimalarial injection & 253 & 35.1 \\
\hline
\end{tabular}

neric form. Antibiotics were combined with antimalarials in 320 prescriptions (44.4\%) and analgesics in 374 prescriptions (51.5\%). Antimalarial drugs were prescribed in injection form for 253 patients $(35.1 \%) ; 18.2 \%$ of those who were prescribed injections (6.4\% of the total) actually had negative blood films for malaria.

The patient care indicators showed that for 718 prescriptions $(99.7 \%)$ the prescribed antimalarial drugs were actually dispensed (Table 3). Chloroquine was prescribed in the great majority of prescriptions. Adequately labelled antimalarials, i.e. the label contained at least the patient name, the drug name and when the drug should be taken, were found in only $80(11.1 \%)$ prescriptions.

When asked to state the dosage schedules of the antimalarial treatment they had received, only 357 patients $(49.6 \%)$ had adequate knowledge of their prescribed treatment, i.e. the drug name, when it was to be taken and in what quantity.

At the time of the study, all the key antimalarial drugs (chloroquine in all formulations, sulfadoxine-pyremethamine tablets, quinine tablets and injections) were found in stock in 7 of the 24 visited health centres. Treatment guidelines in the form of poster information were found in 17 $(70.8 \%)$ health centres (Table 4$)$. 
Table 3 Patient care indicators in health

facilities of Khartoum state $(n=720$ prescriptions/patients)

\begin{tabular}{|c|c|c|}
\hline Patient care indicator & No. & $\%$ \\
\hline $\begin{array}{l}\text { Prescriptions where antimalarial } \\
\text { actually dispensed }\end{array}$ & 718 & 99.7 \\
\hline $\begin{array}{l}\text { Prescriptions where antimalarial } \\
\text { adequately labelled }\end{array}$ & 80 & 11.1 \\
\hline $\begin{array}{l}\text { Patients with adequate } \\
\text { knowledge of prescribed dose }\end{array}$ & 357 & 49.6 \\
\hline
\end{tabular}

a Label shows at least patient name + drug name + when the drug should be taken.

${ }^{b}$ Knowledge of drug name + when to be taken + in what quantity.

\section{Discussion}

Prompt and accurate diagnosis of malaria is the key to effective disease management, and reducing the unnecessary use of antimalarial drugs [9]. Health care workers should make a proper assessment for appropriate management. This not only requires clinical skills but also sufficient consultation time [10]. Many patients in this study were not physically examined, reflecting a deviation from the standard way of managing patients. Heavy patient loads in some health centres, poor knowledge and poor clinical skills or disinterest of the prescriber may be associated with such practices. Lack of close supervision and lack of proper training may also contribute. This could have

Table 4 Health facility indicators in Khartoum state ( $n=24$ health centres)

\begin{tabular}{lcc}
\hline Health facility indicator & No. & $\%$ \\
\hline $\begin{array}{l}\text { Facilities with all key } \\
\quad \begin{array}{l}\text { antimalarial drugs in stock } \\
\text { a }\end{array}\end{array}$ & 7 & 29.2 \\
$\begin{array}{l}\text { Facilities with treatment } \\
\text { guidelines poster available }\end{array}$ & 17 & 70.8 \\
\hline
\end{tabular}

${ }^{a}$ Availability was assessed against a predetermined list of 6 forms of key antimalarial drugs recommended by the national treatment guidelines. a negative impact on patients' satisfaction with the care provided in health centres, and may enhance self-medication, as many patients may skip doctor's consultations and go straight to pharmacies to get their medicines [11]. However, when cases of malaria are treated on the basis of clinical diagnosis this leads to overdiagnosis of malaria and overuse of antimalarial drugs [10]. Efforts have been made to develop guidelines, such as the integrated management of childhood illnesses (IMCI), to assist health workers in such settings.

In areas of intense malaria transmission, where the burden of malaria is greatest and where severe disease and mortality are largely confined to children under 5 years of age, malaria treatment is often dispensed on the basis of "fever" rather than on the basis of a parasitologically confirmed diagnosis. However, with high malaria transmission and high levels of immunity, a significant proportion of the infections are asymptomatic, and detecting parasites in the blood does not always help to distinguish malaria from other causes of fever. Moreover, in most of these areas microscopy and rapid diagnostic tests (RDTs) are not generally available at the periphery of the health services or at the community level, where most cases of malaria are managed. In 2004, a WHO technical consultation recommended microscopy and RDTs for parasitological confirmation of malaria. In all settings, laboratory services that provide malaria microscopy should be strengthened. Where microscopy is not possible, RDTs should be introduced [9]. Some patients in this study were prescribed antimalarial drugs even though parasites were not detected in their peripheral blood films. Higher figures were shown in a study from Tanzania; more than $60 \%$ of those diagnosed with malaria were negative for malaria parasites [10]. 
Factors related to both patients and prescribers may be responsible for prescribing antimalarial drugs for malaria-negative patients. Some prescribers tend to rely on their limited experience as they do not trust laboratory results and prefer treating malaria cases on the basis of clinical criteria. This attitude may be based on fear of losing patients' confidence. Another reason may be that in many developing countries such as Sudan, microscopy is not reliable because microscopists are insufficiently trained and supervised or are overworked, the microscopes and reagents are of poor quality and often the supply of electricity is unreliable [12]. On the other hand, patient demand may be another factor: some patients may not be willing to put up with even minor physical discomfort, and are difficult to convince that they do not have malaria and do not need any treatment [11].

To produce the desired effect, antimalarial drugs need to be given in adequate dosages. However, in our study, the dosage was written in a correct and informative manner in only $60.0 \%$ of prescriptions. This alarming figure indicates a major problem regarding antimalarial dosages, and ultimately would have serious health consequences for the population as under-dosage, together with noncompliance, is one of the reasons for the persistent presence of $P$. falciparum malaria in the population [13].

Our study also showed a high rate of prescribing antibiotics with antimalarial drugs. More than half of malaria-negative patients were prescribed antibiotics with antimalarials. In some situations the misuse of antibiotics is often due to uncertainty about the diagnosis, as the clinical picture is not clear or may even be misleading. Physicians tend to overestimate the severity of illness to justify antibiotic prescribing. They are also under pressure from patients seeking rapid amelioration of symptoms [14].
Prescribing analgesics with antimalarial drugs is also a widespread practice. The patient's temperature could be lowered by mechanical antipyresis, e.g. fanning and sponging down with tepid water. These methods are particularly important for young children, and can help in bringing down the temperature even without giving an antipyretic. Routine antipyretic therapy in children with malaria has long been a source of controversy. However, the usefulness of this practice has not been proven. In a randomized clinical trial conducted in Gabon, it was found that paracetamol had no antipyretic benefits over mechanical antipyresis alone in $P$. falciparum malaria. Moreover, it was found that paracetamol prolonged parasite clearance time, possibly by decreasing production of tumour necrosis factor and oxygen radicals [15].

Another common type of irrational antimalarial use is overprescribing of injections. Injections are an important, powerful and useful formulation when prescribed and used properly, but potentially harmful and dangerous when prescribed carelessly [16]. Furthermore, injections are more expensive than oral forms of drugs. In many countries, as in Sudan, some prescribers and patients believe that injections have a stronger effect and work faster than oral antimalarial drugs. In our study, the highest rate of injection prescriptions was among adult patients, and $18.2 \%$ of those who were prescribed injections actually had negative blood films for malaria. The overprescribing of injections, as explained by other authors, is mainly due to lack of appropriate training of health workers, lack of public education and patients' demands and beliefs [16].

Dispensing is an important step in ensuring antimalarial drug accessibility, affordability, safety and rational use. Almost all prescribed antimalarial drugs in the study were actually dispensed. High rates of dis- 
pensing at the health centres can contribute to patient trust and cooperation with the health system and personnel [14]. However, it was evident that the key antimalarial drugs were not always in stock. Chloroquine was available in all formulations in all health centres, with the exception of 1 health centre in which chloroquine tablets were out of stock. In that health centre the rate of prescription of injections increased, reaching more than $70 \%$. One way to promote rational use is by ensuring that all essential antimalarial drugs are available on a regular basis.

The results also showed poor patient knowledge about the treatment they were prescribed. This raises the question whether the information about prescribed antimalarial drugs was offered voluntarily by health providers or whether they expected patients to ask questions. Improving the physical layout of health centres (examination and dispensing areas) to facilitate patientprovider communication may help improve patients' understanding of prescribed antimalarial drugs [8]. Antimalarial drug labelling is one way to improve patient's adherence to treatment. It reinforces the information given by the prescriber and dispenser, and it should be in clear and simple language. Our results showed poor labelling practices in many health centres. In many cases the patient's name was missing and dosage schedules were incomplete or sometimes incorrect. Lack of knowledge about labelling on the part of the dispensers may have accounted for this finding.

In Sudan, the national treatment guidelines are considered very useful in providing guidance to health professionals. It was evident that the level of knowledge about paediatric doses of antimalarials among prescribers and dispensers was average. Calculating paediatric doses is a genuine problem. It is not always easy for a prescriber with limited skills to calculate the dose in milligrams, and then to change it into millilitres in order to write down the prescription in a correct, simple and understandable form. It is the responsibility of the prescriber to ensure that the dose is accurately calculated, and this responsibility should not be shifted to the dispenser. Poor knowledge will in turn result in poor prescribing practices and ineffective treatment. However, only $23.8 \%$ of prescribers and $44.4 \%$ of dispensers were knowledgeable about the guidelines, i.e. knew the correct malaria case definition and knew different lines of treatment for uncomplicated malaria. Some claimed that the guidelines were not suitable for treating all cases of uncomplicated malaria. Some suggested an increase in the number of tablets. Others thought that 2 injections per day caused hypotension, and suggested that the injectable dose be reduced. On the other hand, some suggested that new antimalarial drugs be added. Considering that effective treatment is the main national control strategy for malaria in Sudan, there is a need to determine the extent to which health care providers follow guidelines. It was found that only $38.6 \%$ of the total number of patients were actually managed according to guidelines. Extensive and continuous training is needed to improve health providers' knowledge and hence promote adherence to national treatment guidelines.

In conclusion, this study showed inappropriate prescribing practices and consequently inappropriate use of drugs for malaria. Similar results have been shown in other developing countries $[2,3,17]$. As Sudan has recently changed the national antimalaria treatment from monotherapy (chloroquine) to artemisinin-based combination therapy, the results of this study should be taken into consideration for improving prescribing and dispensing practices. 


\section{References}

1. Malik EM, Khalafalla OM. Malaria in Sudan: past, present and the future. Gezira journal of health sciences, 2004, 1(Suppl.):47-53.

2. Abdo-Rabbo A. Patients' care regarding consultation and dispensing in some of the public hospitals and private clinics in Sana'a. INRUD news, 1995, 2(5):22-3.

3. Abdo-Rabbo A. Baseline prescribing and health facility indicators in Yemen. Journal of the Faculty of Medicine, Baghdad, 2000, 42(4):824-9.

4. Laing R, Hogerziel H, Ross-Degnan D. Ten recommendations to improve use of medicine in developing countries. Health policy and planning, 2001, 16(1):13-20.

5. Penelope A et al. Diagnostic and prescribing practices in peripheral health facilities in rural western Kenya. American journal of tropical medicine and hygiene, 2003, 68(4 Suppl.):44-9.

6. George KV et al. A study on drug prescribing pattern in Madurai city. Indian journal of pharmacology, 2002, 34:361-2.

7. Haak H. Access to antimalarial medicines. Improving the affordability and financing of artemisinin-based combination therapy. Geneva, World Health Organization, 2002 (WHO/CDS/MAL/2003.1095).

8. How to investigate drug use in health facilities (selected drug use indicators). Geneva, World Health Organization, 1993 (WHO/DAP/93.1).

9. The role of laboratory diagnosis to support malaria disease management. Focus on the use of rapid diagnostic tests in areas of high transmission. Report of a WHO technical consultation 25-26 October 2004. Geneva, World Health Organization, 2006 (WHO/HTM/MAL/2006.1111).
10. Nsimba SED et al. Case management of malaria in under-fives at primary health care facilities in a Tanzanian district. Tropical medicine and international health, 2002, 7(3):201-09.

11. De Vries TPGM et al. Guide to good prescribing: a practical manual. Geneva, World Health Organization, 1994 (WHO/ DAP/94.11).

12. Ibrahim SM. Decisive assessment of diagnostics staining methods of malaria in eight public and private laboratories, Khartoum area. In: Operational research in tropical diseases, final report summaries 1992-2000. Cairo, World Health Organization Regional Office for the Eastern Mediterranean, 2003 (WHO-EM/ TDR/004/E/G)

13. Jimmy EO, Achelonu E, Orji S. Antimalarial dispensing pattern by patent medicine dealers in rural settlements in Nigeria. Public health, 2000, 114(4):282-5.

14. Otoom $S$ et al. Evaluation of drug use in Jordan using WHO prescribing indicators. Eastern Mediterranean health journal, 2002, 8(4/5):537-43.

15. Brandts $\mathrm{CH}$ et al. Effect of paracetamol on parasite clearance time in Plasmodium falciparum malaria. Lancet, 1997, 350:704-9.

16. Abdo-Rabbo A. Prescribing rationality and availability of antimalarial drugs in Hajjah, Yemen. Eastern Mediterranean health journal, 2003, 9(4):607-17.

17. Hogerzeil HV. Promoting rational prescribing: an international perspective. British journal of clinical pharmacology, 1995, 39(1):1-6. 